\title{
Quality of life issue in psoriasis: A study from western Nepal
}

\author{
Kumar A, ${ }^{1^{*}}$ Neupane $S,{ }^{2}$ Shrestha PR, ${ }^{1}$ Pun $J,{ }^{1}$ Thapa , $^{1}$ Manandhar $M^{1}$ \\ ${ }^{1}$ Department of Dermatology, Manipal College of Medical Sciences, Pokhara. \\ ${ }^{2}$ Department of Dermatology, Gandaki Medical College, Pokhara.
}

*Corresponding Author:

Dr Ajay Kumar,MD

Assistant professor,

Department of Dermatology.

Manipal College of Medical Sciences, Pokhara Nepal.

E-mail: drajayk2002@yahoo.com

\begin{abstract}
Background: Psoriasis may have negative impact on a patient's quality of life. Very few studies have examined this aspect in Nepalese population. Aim of this study was to evaluate the Quality of life (QOL) in chronic psoriatic patients by modified version of psoriasis disability index (PDI).

Methods: Present cross sectional study was conducted in three major hospitals in western Nepal. One hundred chronic psoriatic patients of 18 years or more of age, with disease duration of six months or more and body surface area involvement of $10 \%$ or more were recruited. Clinical assessment was done with psoriasis area severity index (PASI) and quality of life was measured by modified version of PDI. Patients were managed as per indication. At the follow up at four weeks, improvement in PASI and improvement in QOL was measured. Statistical analysis by Chisquare test was done to assess the relation between severity of psoriasis and different items of quality of life.
\end{abstract}

Results: Psoriasis has negative impact on all aspect of quality of life in Nepalese patients, as shown by score of modified version of PDI, which was $39.27 \%$ of total. There was significant improvement in PASI at follow up visit, from $23.29 \%$ to $15.35 \%$ of total. There was significant improvement in quality of life after treatment (modified version of PDI) from $39.27 \%$ to $32.66 \%$ of total.

Conclusions: Psoriasis has negative impact on all aspect of quality of life in Nepalese patients.

Key words: Psoriasis; PASI; Modified version of PDI; and QOL.

\section{Introduction:}

Psoriasis, a chronic skin disease affects it varied from $0.91 \%$ to $8.5 \%$ of population, and one of the common causes of hospitalization in dermatology. ${ }^{1}$ It is a multifaceted condition having broad impact on patient's daily life. Psoriasis is associated with cosmetic disfigurement and may be experienced as socially stigmatization and patient may feel flawed, embarrassed, self-conscious and rejected. ${ }^{2,3}$
The traditional measures of psoriasis severity, like PASI, do not provide direct measurement of the impact of the disease as a result, QOL has emerged as an important measure of outcome of therapeutic interventions and there is growing awareness among stake holders that the psychological and occupational impact are as important as physical parameters. $^{3}$ The concept of QOL was developed from information about holistic well-being and was foisted by the World Health Organization's (WHO) broad view of 
health as not merely absence of disease, but the ability of a person to lead a productive and enjoyable life. ${ }^{4}$ It has been agreed that patients are in the best position to assess their QOL.

\section{Methods:}

This was a multi-center study conducted in Manipal Teaching Hospital, Gandaki Medical College Teaching Hospital and Fishtail Hospital and Research Center, Pokhara between 1st May 2012 to 1st May 2014. The study group consisted of 100 consenting consecutive cases of psoriasis of sexes, 18 years or more of age with duration of disease six months or more with body surface area involvement $10 \%$ or more with absence of severe mental or other physical illness. The extent of clinical severity was assessed by PASI. Original version of PDI was modified and translated in Nepali.

At first visit, after verbal consent patient were asked to fill up the questionnaire, illiterate patients were helped by treating physician, clinical severity was measured by PASI. Information on socio-demographic variables, clinical features, and other factors of interest were also collected. Patients were treated as per indication and were requested for follow up in four weeks. At the follow up PASI was recorded and another sheet of modified version of PDI was filled up.

\section{Statistical analysis:}

For both the PASI and modified version of PDI, paired t-test was used to evaluate the difference between scores at first visit and at follow-up. Chi-square test was used to examine the relation between severity of psoriasis and different items of QOL. All statistical analysis was carried out using SPSS, version 16.0 for Windows.

\section{Results:}

\section{General description of study subjects}

Total numbers of 88 patients completed the study. Female to male ratio was $1.04: 1$. Patients were 18 to 60 years of age. Eighty eight percent (78/88) of the subjects were married. Positive family history of psoriasis was found in (14/88) $15.9 \%$ of the patients (first degree relatives having psoriasis). Twenty one (23.9\%) were illiterate, 45 (51.1\%) were educated up to primary level (fifth grade), 21 (23.9\%) were up to secondary level (tenth grade) and only one had higher education (SLC and above) Duration of illness ranged from six months to 32 years. Itching was a significant problem in $(82 / 88) 93.2 \%$ of the patients. Similarly troublesome scaling was a problem in (83/88) $94.3 \%$ of the patients. Aesthetic disfigurement was reported by (51/88) $58 \%$ of the patients.
Impact of psoriasis on quality of life was studied in relation to six domains: daily activities work or school/ alternative questions if not at work or school, personal relationship, leisure, treatment and others.

Daily activities: it included four items. In about $90 \%$ of the patient psoriasis interfered with carrying out work around the house or garden, and almost all the patients reported that they had to worn different types/colors of clothes, had to change/wash clothes more frequently, and had to bath more often than usual because of psoriasis. Overall, psoriasis affected all the four items of daily activities significantly.

Work or school/ alternate questions if not at work or school: It included three items. Almost $90 \%$ of the patients reported that they lost time from work or school/ the disease stopped carrying out normal daily activities and the disease prevented them doing things at work or school/ altered the way they carry out their normal daily activities, career was affected in almost $90 \%$ of patients.

Personal relationship: It included four items. About $40 \%$ of the patients had their sexual activities affected either a little or a lot and about $70 \%$ reported that their psoriasis had created problems with husband wife relationship. Almost $90 \%$ of these patients had some problem with their relatives or community members because psoriasis.

Leisure: It has three items. About $90 \%$ of the patients reported that psoriasis stopped them going out socially. About $80 \%$ of the patients reported that they were unable to use, criticized from using community water resources/ bathing and about $55 \%$ reported that the disease had resulted in smoking or drinking alcohol more than they would do normally.

Treatment: It included two items. More than $85 \%$ of these patients reported that the treatment had made their home messy or untidy and it is costly.

Others: Six items were included in this domain and were related to personal and social stigma and the frequent questions asked by the patients to their physician, regarding the nature of psoriasis, is it contagious? Is it related with leprosy? Is it related with cancer? About $53 \%$ of the patients thought that the disease was associated with leprosy and $70 \%$ reported that their relatives/ community member think it is associated with leprosy. About $56 \%$ of the patients thought that the condition is contagious, whereas about $90 \%$ of their relatives/ community members thought the condition is contagious. About $65 \%$ of the patients related psoriasis with cancer, and about $80 \%$ of their relatives/ community members related it with cancer.

\section{Impact of psoriasis on quality of life}


Descriptive information of PASI and modified version of PDI scores at first and follow up visit are shown in Table 1.

Table1: PASI and modified version of PDI scores at first and follow up visit.

\begin{tabular}{|l|c|c|c|c|}
\hline & Min. & Max. & Mean & $\begin{array}{c}\text { Std. } \\
\text { Deviation }\end{array}$ \\
\hline PASI at $1^{\text {st }}$ visit & 9.00 & 44.10 & 16.77 & 7.19 \\
\hline PASI at $2^{\text {nd }}$ visit & 2.40 & 32.10 & 10.98 & 5.24 \\
\hline${\text { Modified PDI at } 1^{\text {st }} \text { visit }} 13$ & 43 & 25.92 & 6.98 \\
\hline${\text { Modified PDI at } \mathbf{2}^{\text {nd }} \text { visit }}$ & 2 & 34 & 21.56 & 6.89 \\
\hline
\end{tabular}

Score of different items of modified version of PDI at first visit is shown in figure 1 . No significant correlations was found between age of the patients and impairment in quality of life $(p=.801, r=-0.027)$. Similarly there was no correlation between duration of illness and impairment in QOL $(\mathrm{p}=0.830, \mathrm{r}=.023)$.

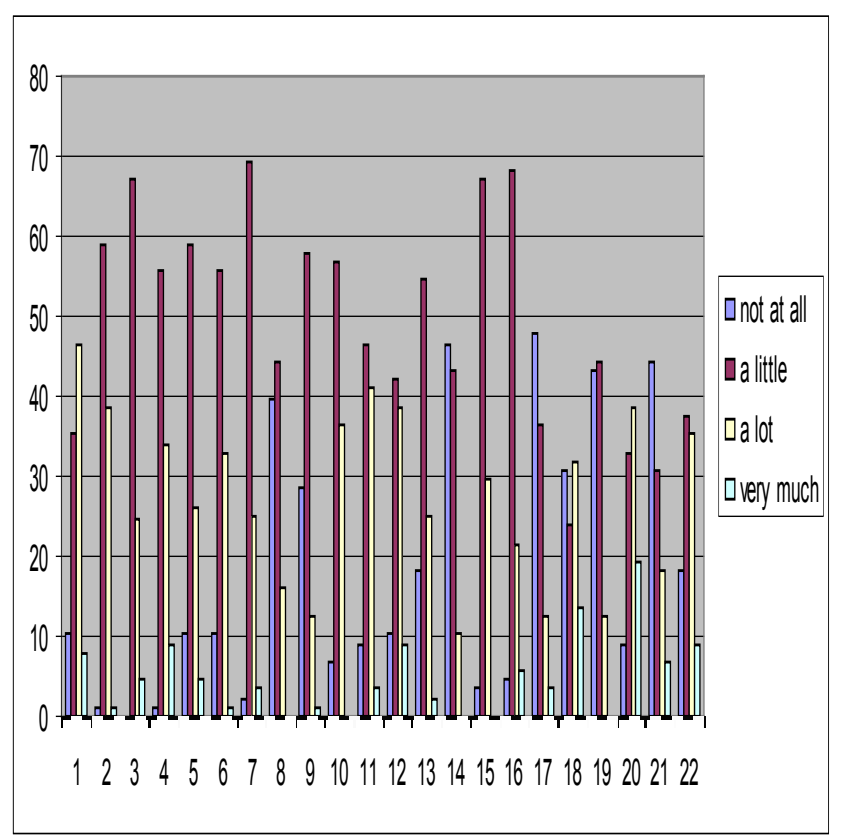

Fig. 1: Bar diagram showing score of different items of modified version of PDI at first visit.

1. Interfered with carrying work around the house or garden

2. Wore different types or colors of clothes.

3. Frequently changed or washed clothes.

4. Had to take more than usual baths

5. Lost time from work or school/ prevented carrying out normal daily activities.

6. Prevented doing things at work or school/ altered the way in which normal daily activities carried out.

7. Career affected.

8. Sexual difficulties
9. Problems with husband wife relationship.

10. Problems with relatives.

11. Problems with community members

12. Stopped going out socially or to any other social functions.

13. Unable to use,criticized or stopped using communal bathing facilities.

14. Smoking or drinking alcohol more than usual.

15. Treatment made home messy or untidy.

16. Treatment is costly.

17. Patient correlates his disease with leprosy

18. Community members/relatives correlate the disease with leprosy.

19. Patient thinks his disease is contagious.

20. Community members/ relatives think that the disease is contagious.

21. Patient correlates his disease with cancer.

22. Community members/ relatives correlate the disease with cancer

\section{Discussion:}

Skin is responsible for self-image and influences others' perception of us. When this organ is disfigured, the impact on persons' self-image, and how he or she is seen by others, can be severe. ${ }^{6}$ Quality of life has emerged as an important outcome of patient care, and there has been growing interest in its measurement in skin disease. ${ }^{7}$ It has been reported that disability experienced by psoriasis sufferers are comparable to that of other chronic illness like heart disease, diabetes and depression. ${ }^{2}$

In present study, among all domains, daily activities were affected the most $(>90 \%)$ which are comparable to previous studies. ${ }^{5,8,9}$ Compared to Al- Mazeedi et al ${ }^{10}$ where it was only $50 \%$. Psoriasis has negative impact on work or occupation. In present study about $90 \%$ had their work, career or houses hold activities affected due to psoriasis. Similar findings were reported by Finlay AY and Coles EC. ${ }^{5}$ compared to Al-Mazeedi et $\mathrm{al}^{10}$ where it was $17.1 \%$ and in another study psoriasis didn't influence the choice of career. ${ }^{11}$ About $40 \%$ of patients of our study had their sexual life affected a little or a lot, and in $70 \%$ it had affected their relationship with spouse. Similar figure ( $>40 \%)$ was reported by Gupta MA and Gupta AK. ${ }^{12}$ and Al-Mazeedi et al. ${ }^{10}$ In present study about $90 \%$ patients stopped going out socially and about $80 \%$ felt discriminations at communal places. Al-Mazeedi et al ${ }^{10}$ reported that $48.2 \%$ of cases stopped making friends and meeting people due to psoriasis, and two thirds worry about the thoughts and reactions of others towards their disease. Similar results were reported by Krueger $\mathrm{G}$ et $\mathrm{al}^{13}$ and Vardy $\mathrm{D}$ et al. ${ }^{14}$ In present study 
about $55 \%$ of patients had resulted drinking alcohol or smoking more than they were doing. Similarly Zachariae et a ${ }^{15}$ revealed weak, but statistically significant associations between both disease severity and impairment of QOL and alcohol consumptions and smoking. These behaviors could be interpreted as difficulties in coping with psoriasis and subsequent reduction in QOL. In present study treatment was costly and was responsible for making untidy home in more than $85 \%$ of the patients. In present study, psoriasis was thought to be contagious in $56 \%$ of the patients and $90 \%$ of their relatives or community members. About $53 \%$ of the patient and $70 \%$ of their relatives correlated psoriasis with leprosy and $60 \%$ of patient and $80 \%$ of their relatives correlated psoriasis with cancer. Similarly Vardy D et al ${ }^{14}$ reported that psoriatic patients are socially withdrawn due to ugliness and fear of contagious conditions.

Score of modified version of PDI at first visit was $39.27 \%$ of total. Similar results were reported by Finlay and Coles ${ }^{5}$ (38.8), Wahl et $\mathrm{al}^{16}$ (35 and 33.2) whereas, Dubertret et al ${ }^{17}$ and Zachariae et $\mathrm{l}^{15}$ found lower mean PDI (21 and 24.0). However, in Chinese patients by Yang $\mathrm{Y}$ et al ${ }^{18}$ clinical severity was not associated with psychological and PDI scores. Heydendael et $\mathrm{al}^{19}$ concluded that psoriasis patients had a lower QOL than reference population, without a significant relation between disease severity and QOL. Kirby et $\mathrm{al}^{18}$ found that clinical psoriasis severity measurement like PASI provide only a partial indication of the psychological disability and physical assessment scores do not reflect psychological disability. In Nordic patients PASI was not significant predictor of QOL even though the PASI scores did correlate with self-reported severity. ${ }^{16}$ A literature review on relationship between disease severity and QOL by Korte et $\mathrm{al}^{20}$ found weak association. It has been said that changes in clinical severity reflect the biologic relevance of an intervention, whereas, QOL measure quantify how those changes impact on the daily life. ${ }^{21}$

Although the severity of psoriasis is of significance, it is important to document what the patient perceives as most bothersome aspect of their psoriasis and its management and what their hopes and expectations are from physicians. This knowledge would simplify formulation of a treatment plan with more appropriate individual goals.

Cross-sectional design did not permit us to examine a possible sequential relationship between psychological distress and psoriasis. Moreover, the sample was restricted to tertiary clinic attendees, which may not fully represent the population of psoriasis. While measuring the impact of psoriasis on QOL no control group were used.

\section{Conclusions:}

Psoriasis has negative impact on all aspect of quality of life in Nepalese patients. Management should consider both clinical parameters as well as the factors affecting QOL. When the negative impact of the disease is more, management should be aggressive. To decrease the stigmatization, greater effort is needed to raise awareness that psoriasis is not contagious, but is a disease like many other chronic conditions.

\section{References:}

1. Parisi R, Symmons DP, Griffiths CE, et al. Global epidemiology of psoriasis: a

systematic review of incidence and prevalence. J Invest Dermatol 2013;133:377-85.

2. Rapp SR, Feldman SR, Exum ML, et al. psoriasis causes as much disability as other medical diseases. J Am Acad Dermatol 1999;41:401-7.

3. Hrehorów E, Salomon J, Matusiak L, et al. Patients with psoriasis feel stigmatized. Acta Derm Venereol. 2012;92:67-72.

4. World health Organization. International classification of Impairments, Disabilities and Handicaps, Geneva: World Health Organization, 1980;27-29.

5. Finlay AY, Coles EC. The effect of severe psoriasis on the quality of life of 369 patients.Br J dermatol 1995;132:236-44.

6. Russo PAJ, Ilchef R, Cooper AJ. Psychiatric morbidity in psoriasis: A review. Aus J Dermatol 2004;45:155-61.

7. Kirby B, Fortune DG, Bhusan M, et a.l The Salford Psoriasis Index: a holistic measure of psoriasis severity. Br J Dermatol 2000;142;728-32.

8. Rakhesh SV, D'Souza M, Sahai A. Quality of life in psoriasis: A study from south India. Indian J Dermatol Venereol Leprol. 2008;74:600-6.

9. Manjula VD, Sreekiran S, Surendran S, et al. Study of psoriasis and quality of life in a tertiary care teaching hospital of kottayam, kerala . Indian J Dermatol. 2011 Jul;56:403-6.

10. Al-Mazeedi K, El-Shazly EL and Al- Azmi HS. Impact of psoriasis on quality of life in Kuwait. Int $\mathbf{J}$ Dermatol.2006;45:418-32.

11. Ramsay B, O'Reagan MA. Survey of the social and psychological effects of psoriasis. $\mathrm{Br} \mathrm{J}$ Dermatol 1998;118:195-201. 
12. Gupta MA, Gupta AK. Psoriasis and sex: a study of moderately to severely affected patients. Int J Dermatol 1997;36: 587-91.

13. Krueger G, Koo J, Lebwohl M, et al. The impact of psoriasis on quality of life: result of a 1998 National Psoriasis foundation patient-membership survey. Arch Dermatol 2001;137:280-4.

14. Vardy D, Besser A, Amir M, et al. Experiances of stigmatization play a role in mediating the impact of disease severity on quality of life psoriasis patients. $\mathrm{Br}$ J Dermatol 2002;147:736-42.

15. Zachariae R, Zachariae H, Blomqvist K et al. Quality of life in 6497 Nordic patients with psoriasis. Br J Dermatol 2002;146:1006-16.

16. Wahl A, Loge JH, Wiklund I et al. The burden of psoriasis: a study concerning health related quality of life among Norwegian adults with psoriasis compared with general population norms. J Am Acad of Dermatol 2000;43:803-8.
17. Dubertret L, Mrowietz A, Ranki A et al. Europian patient perspective on the impact of psoriasis: the EUROPSO patient membership survey.Br J Dermatol. 2006;155:729-736.

18. Yang Y, Koh D, Khoo L et al. The psoriasis disability index in Chinese patients: contribution of clinical and psychological variables. Int J Dermatol. 2005;44:925-9

19. Heydendael MRV, Corianne AJM Spulis IP et al. The burden of psoriasis is not determined by disease severity only. J Investig Dermatol 2004; 9: 131-35

20. Korte DJ, Sprangers AGM, Mombers MCF and Bos DJ. Quality of life in patients with psoriasis: a systematic literature review. J Investig Dermatol.2004;9:240-47.

21. Vera MRH, Corianne AJM, Phyllis IS, et al. Jan DB and Menno A. The burden of psoriasis is not determined by disease severity only. J Investig dermatol 2004;9:131-35. 